\title{
DCE/N'T//0872 - T69
}

\section{4th Quarterly Report}

1. Linda D. Stetzenbach, Harry Reid Center for Environmental Studies, University of Nevada, Las Vegas.

2. Identification of Subsurface Microorganisms at Yucca Mountain

3. Bacteria isolated from water samples collected in a series of ground water springs have been isolated, enumerated, and identified from twenty six sites. Ten sites were sampled in Death Valley, California and sixteen sites were sampled in Ash Meadows, Nevada. Replicate samples were collected and tested from four locations. All water samples were collected in conjunction with the HRC Chemistry group conducting ground water fingerprinting studies. The protocol for collection of samples, as described in the 3rd quarterly report, specified aseptic collection in sterile screw-capped containers and transportation on ice to the HRC microbiology laborat $\because$. All samples were inoculated by spread plating onto R2A (Difco Laboratories, Detroit, MI) bacteri..i culture medium. The R2A plates were then incubated at $28^{\circ}$ for 5-7 days and colonies were counted with the aid of a grid template and magnifying lens.

Table 1. Enumeration of hacteria as the number of colony forming units (CFU) per $\mathrm{ml}$ of water collected from sites at Death Valley National Monument, California.

\begin{tabular}{||c|c|c|}
\hline Sample Date & Sample Site & Total CFU/ml \\
\hline 17 March'93 & Grapevine & 190 \\
\hline 16 March'93 & MacClean & 7100 \\
\hline 17 March'93 & Mesquite & 240 \\
\hline 16 March'93 & Navarres & 1080 \\
\hline 18 March'93 & Saratoga & 5550 \\
\hline 17 March'93 & Scotty's Castle & 300 \\
\hline 17 March'93 & Surprise & 1850 \\
\hline 16 March'93 & Texas Springs & 1400 \\
\hline 16 March '93 & Travertine A & 1200 \\
\hline 16 March '93 & Travertine B & 880 \\
\hline
\end{tabular}

\section{DISCLAIMER}

This report was prepared as an account of work sponsored by an agency of the United States Government. Neither the United States Government nor any agency thereof, nor any of their employees, makes any warranty, express or implied, or assumes any legal liability or responsibility for the accuracy, completeness, or usefulness of any information, apparatus, product, or process disclosed, or represents that its use would not infringe privately owned rights. Reference herein to any specific commercial product, process, or service by trade name, trademark, manufacturer, or otherwise does not necessarily constitute or imply its endorsement, recommendation, or favoring by the United States Government or any agency thereof. The views and opinions of authors expressed herein do not necessarily state or reflect those of the United States Government or any agency thereof. 
Table 2. Enumeration of bacteria as the number of colony forming units (CFU) isolated per $\mathrm{ml}$ of water from sites at Ash Meadows Wildlife Refuge, Nevada.

\begin{tabular}{|c|c|c|}
\hline $\begin{array}{c}\text { Sample } \\
\text { Date }\end{array}$ & Sample Site & Total CFU/ml \\
\hline $25 \mathrm{~J}$ an ${ }^{\prime} 93$ & Bradford & 7040 \\
\hline $15 \mathrm{Jan}$ '93 & Cold & 100 \\
\hline 25 Jan '93 & Crystal & 130 \\
\hline $9 \mathrm{Jan}{ }^{\prime} 93$ & Devil's Hole, surface & 50 \\
\hline 9 Jan '93 & Devil's Hole, 20' depth & 130 \\
\hline 9 Jan '93 & Devil's Hole, 40 'depth & 90 \\
\hline $9 \mathrm{Jan}{ }^{\prime} 93$ & Devil's Hole, $85^{\prime}$ depth & 130 \\
\hline $15 \mathrm{Jan}{ }^{\prime} 93$ & Fairbanks & 140 \\
\hline 25 Jan '93 & Forest & 7200 \\
\hline $15 \mathrm{Jan} ' 93$ & Indian & 100 \\
\hline 25 Jan '93 & Kingspool & 7520 \\
\hline 15 Jan 93 & Longstreet & 680 \\
\hline $15 \operatorname{Jan} 93$ & Marsh & 0 \\
\hline 11 Jan 93 & Rogers 1 & 40 \\
\hline $11 \mathrm{Jan}{ }^{\circ} 93$ & Rogers 2 & 50 \\
\hline $11 \mathrm{Jan} ' 93$ & School 1 & 6080 \\
\hline $11 \mathrm{Jan} \cdot 93$ & School 2 & 6000 \\
\hline $15 \mathrm{Jan}{ }^{\prime} 93$ & Scruggs & 230 \\
\hline 11 Jan '93 & Southeast Rocks & 10 \\
\hline $11 \mathrm{Jan} \cdot 93$ & Southwest Rocks & 110 \\
\hline $11 \mathrm{Jan}{ }^{\prime} 93$ & Tubbs 1 & 3400 \\
\hline 11 Jan '93 & Tubbs 2 & 2000 \\
\hline
\end{tabular}


Because environmental isolates may not grow well on nutrient-rich laboratory media, some of the isolates were first cultured on R2A agar and then subcultured to R2A and tryptic soy broth agar (TSBA, Difco) to determine if this procedure would improve the isolation rate. The results shown in Figure 1 indicate the mean percent recovery is about the same on R2A and TSBA. The two results correlate fairly well bui individual values vary widely in mid-range, Figure 2 . This may be due to the response of different species to the growth conditions and needs further investigation.

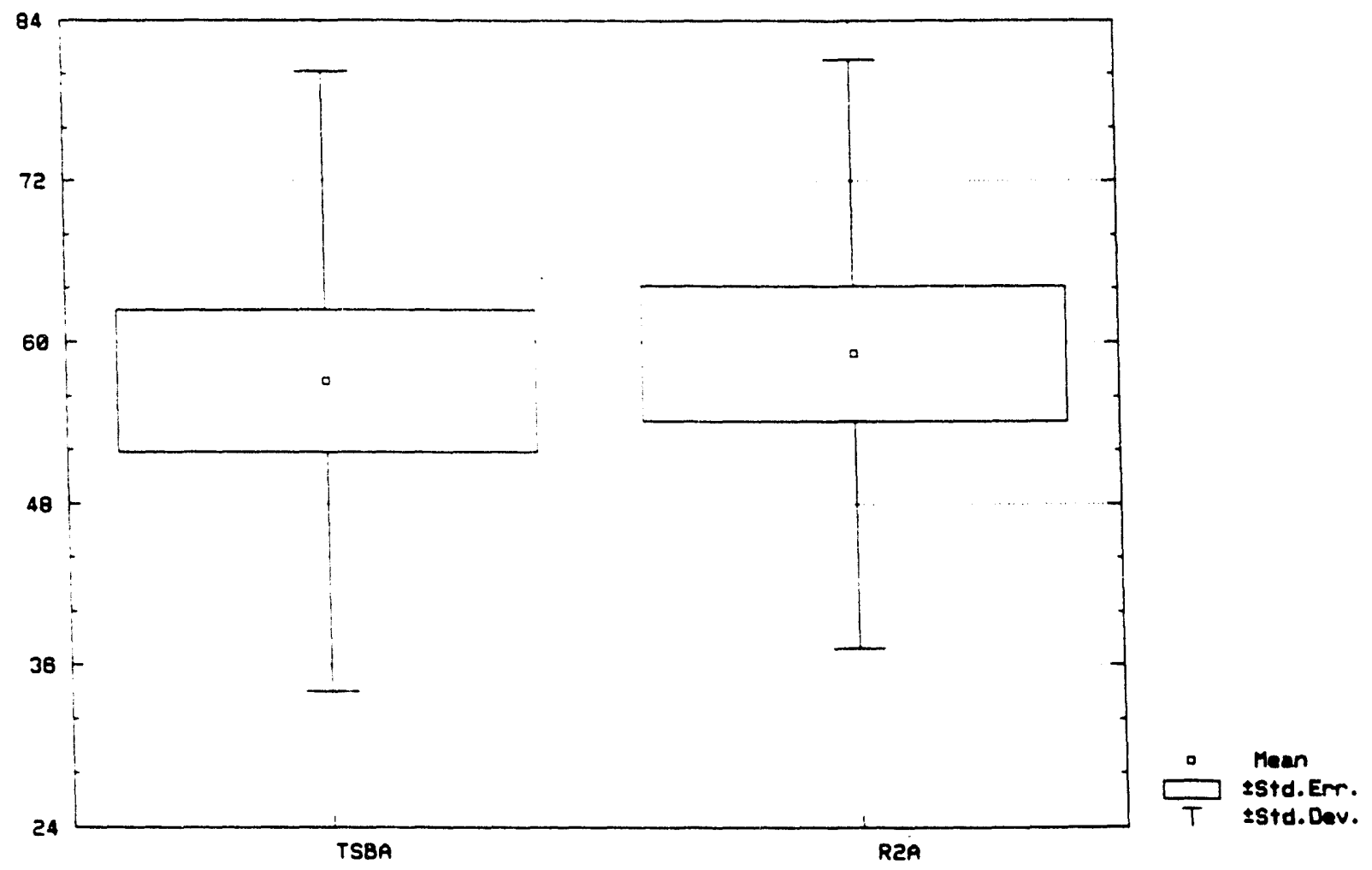

\begin{tabular}{|r|c|c|c|c|c|c|}
\hline & $\mathrm{N}$ & $\operatorname{Min}$ & $\operatorname{Max}$ & Mean & St. Err. & St. Dev. \\
\hline TSBA & 19 & 16.00060 & 97.0000 & 57.10526 & 5.440746 & 23.71566 \\
R2A & 19 & 20.00000 & 100.0000 & 59.15789 & 5.173743 & 22.55182 \\
\hline
\end{tabular}

Figure 1. Comparison of percent recovery of organisms subcultured onto R2A and TSBA. 


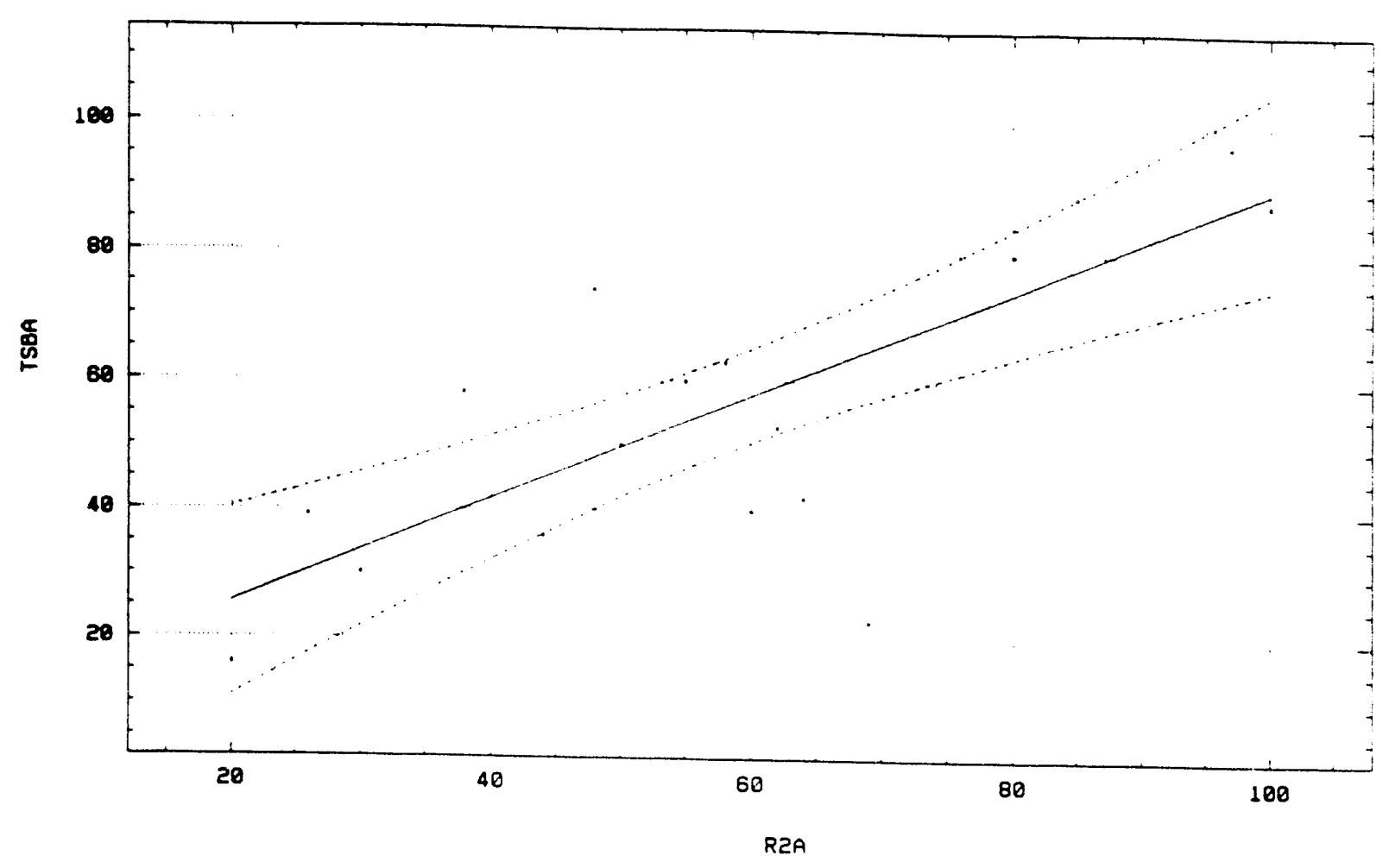

Figure 2. Regression plot of percent recovery of organisms plated onto two media.

Bacterial isolates were processed using the gas chromatographic (GC) based Microbial Identification System, MIDI (Microbial ID, INC., Newark, DE) method. According to the MIDI protocol, bacterial isolates were cultured on tryptic soy broth agar (TSBA, Difco) prior to harvesting. Previous work indicated that about $50 \mathrm{mg}$ wet weight of cells were needed to produce sufficient fatty acid methyl ester peaks to identify an organism. However this rule of thumb seems to be somewhat ciependent on the species and some organisms grow too slowly to accumulate that much mass within a reasonable time. A least squares regression plot of cell mass and peak area was performed for several species to determine the optimum mass for GC analysis. The results show the summed peak area counts for Staphylococcus sp. (Fig. 3), Micrococcus sp. (Fig. 4), and Pseudomonas sp. (Fig. 5) increase as cellular mass increases within the limits of ca. $10 \mathrm{mg}$ to $60 \mathrm{mg}$ wet weight. Bacillus sp. area versus mass plots are almost flat over a broad wet weight range (Fig. 6). Scatterplots of MIDI Similarity Index and mass do not show correlation (data not illustrated).

Identification of isolates using the MIDI system is listed in tables 3 and 4 . Correlation of bacterial isolates with chemical parameters is pending. 


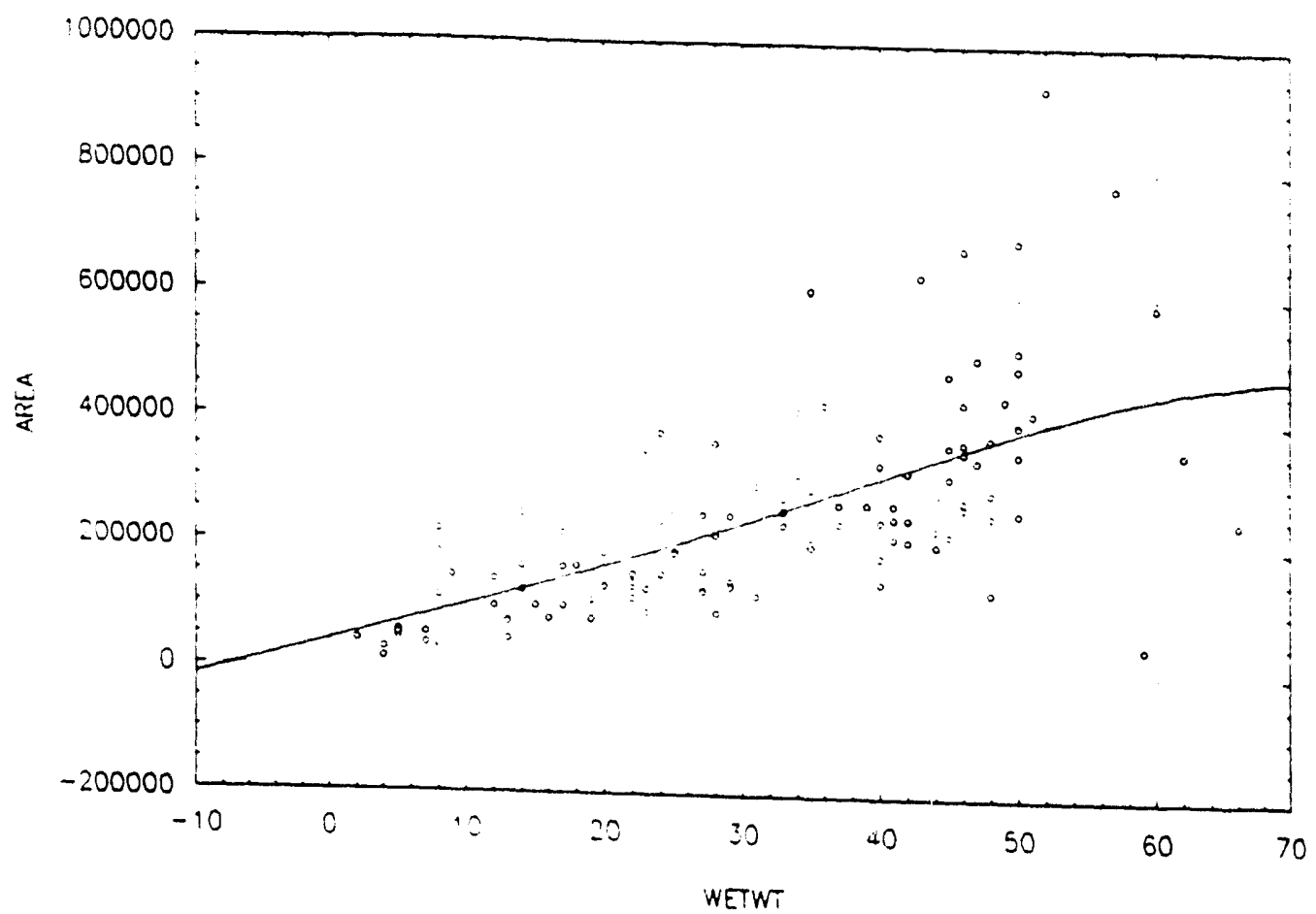

Figure 3. Scatterplot of peak area counts (area) vs. wet weight (wetwt) for isolates identified as Staphylococcus sp. using the MIDI identification system.

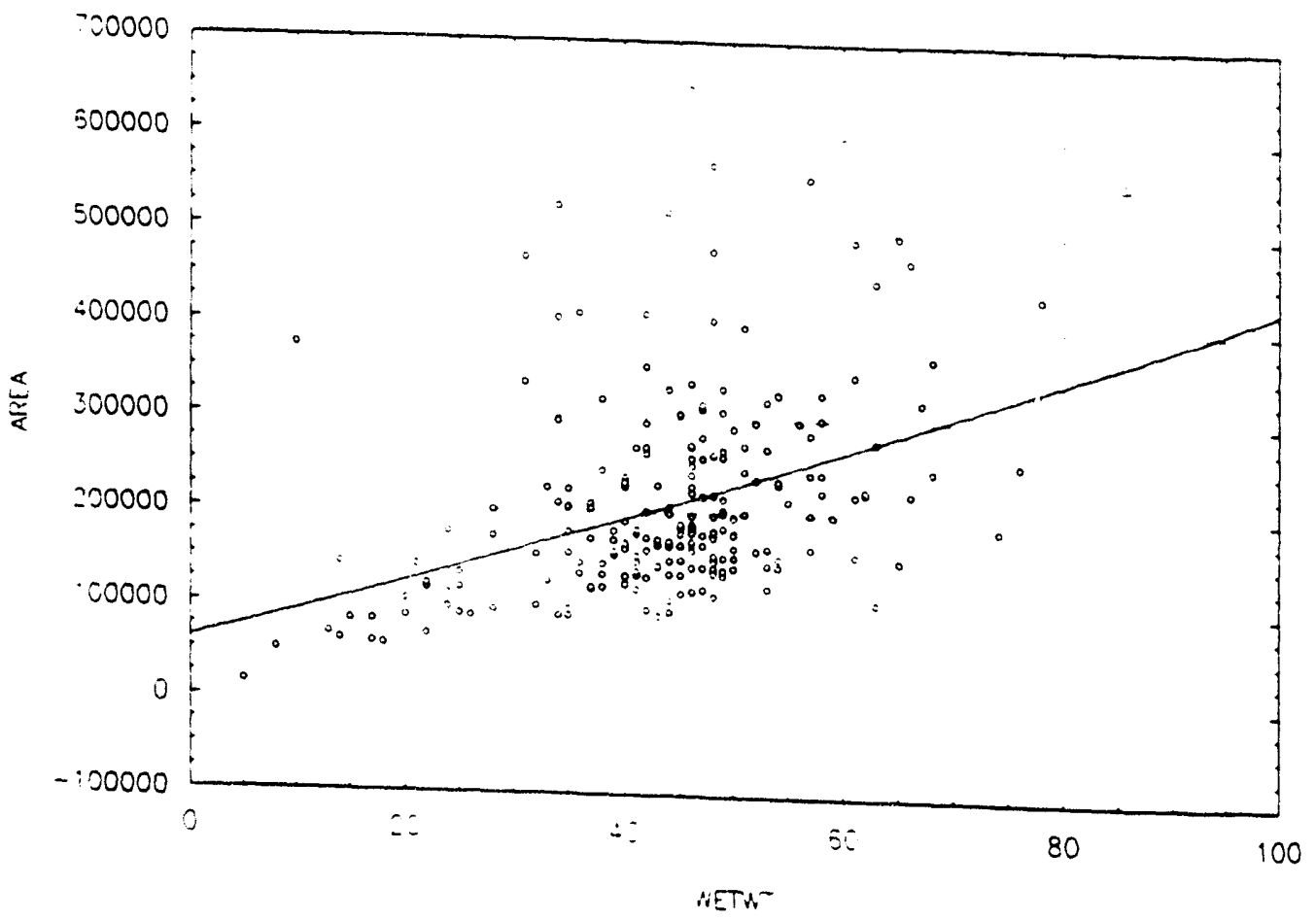

Figure 4. Scatterplot of peak area counts (area) vs. wet weight (wetwt) for isolates identified as Micrococcus sp. using the MIDI identification system. 


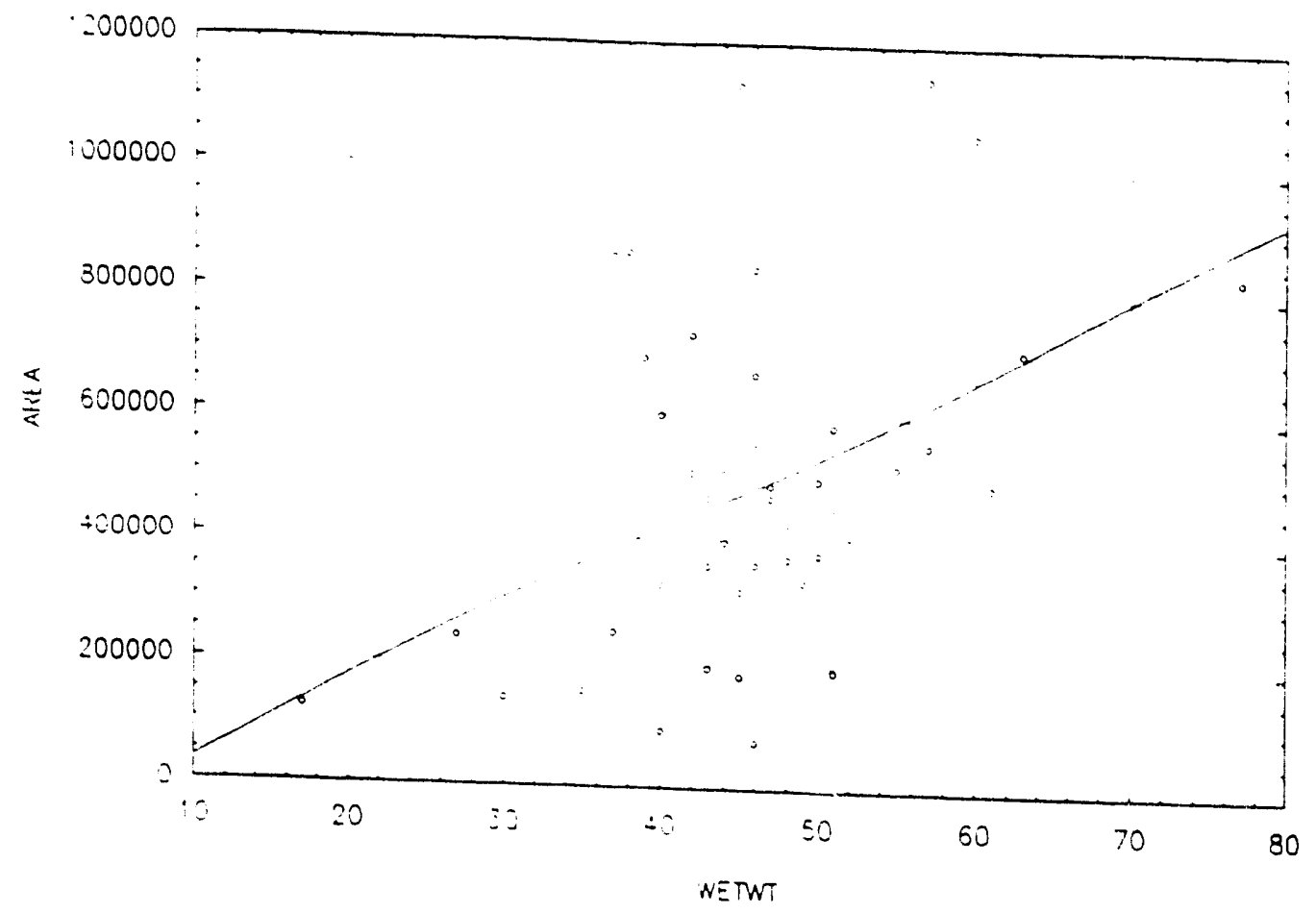

Figure 5. Scatterplot of peak area counts (area) vs. wet weight (wetwt) for isolates identified as Pseudomonas sp. using the MIDI identification system.

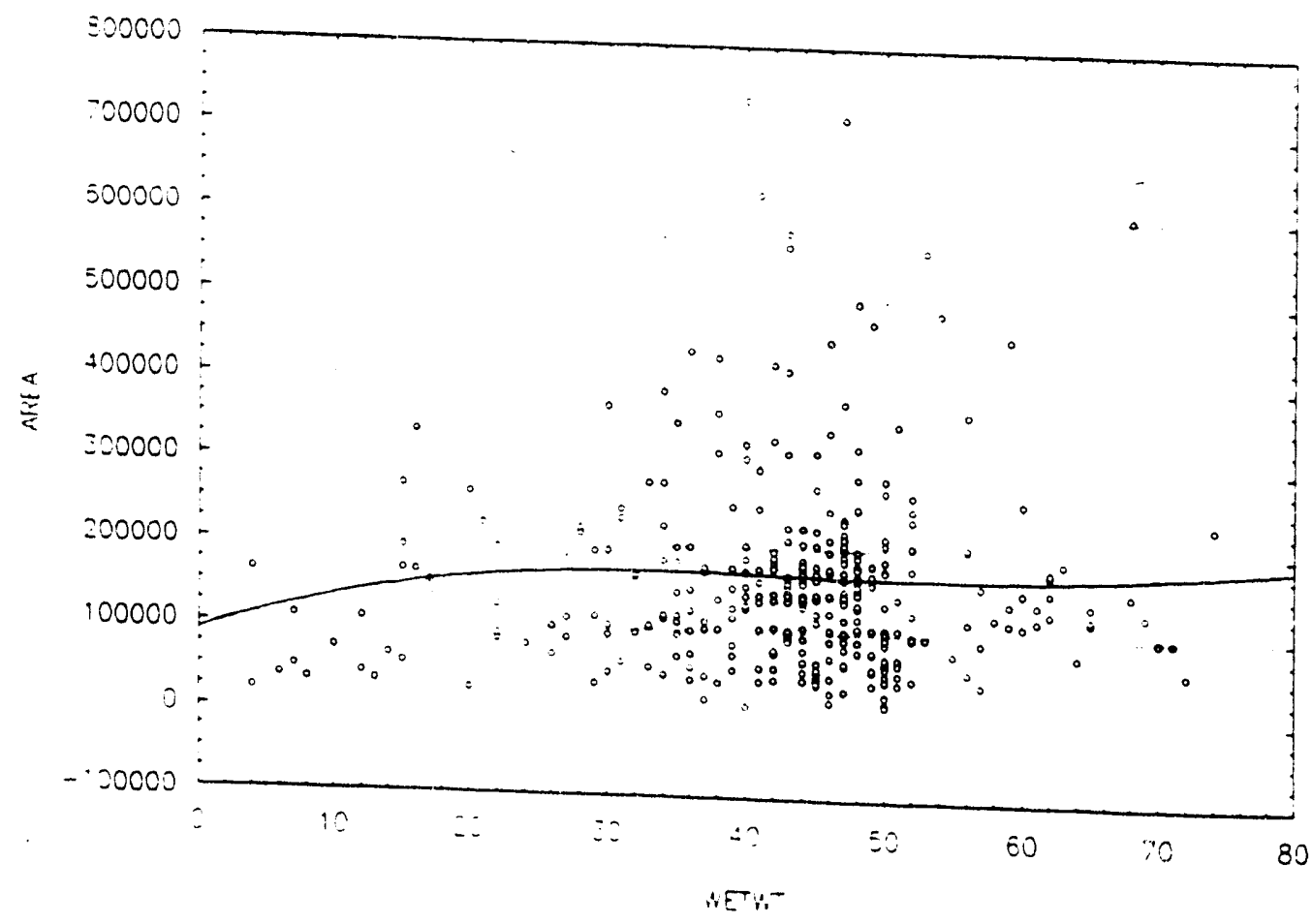

Figure 6. Scatterplot of peak area counts (area) vs. wet weight (wetwt) for isolates identified as Bacillus sp. using the MIDI identification system. 
Table 3. Identification of isolates from the Death Valley National Monument.

\begin{tabular}{|c|c|c|c|c|}
\hline SOURCE & SITE & GENUS1 & SPECIES1 & $10 \times 1$ \\
\hline $\begin{array}{l}D V \\
D V \\
D V \\
D V \\
D V \\
D V \\
D V \\
D V \\
D V \\
D V\end{array}$ & $\begin{array}{l}\text { MACCLEAN } \\
\text { MACCLEAN } \\
\text { MACCLEAN } \\
\text { MACCLEAN } \\
\text { MACCLEAN } \\
\text { MACCLEAN } \\
\text { MACCLEAN } \\
\text { MACCLEAN } \\
\text { MACCLEAN } \\
\text { MACCLEAN }\end{array}$ & \begin{tabular}{|l} 
Bacillus \\
Bacillus \\
Corynebacterium \\
Erwinia \\
Hafnia \\
Methylobacterium \\
Micrococcus \\
Micrococcus \\
No Match \\
Serratia \\
\end{tabular} & $\begin{array}{l}\text { lentus } \\
\text { pumilus sg } 8 \\
\text { mediolamun } \\
\text { salicis } \\
\text { alvei } \\
\text { zatmanii } \\
\text { roseus } \\
\text { roseus } \\
\text { fonticola } \\
\end{array}$ & $\begin{array}{r}12 \\
801 \\
506 \\
828 \\
930 \\
442 \\
614 \\
640 \\
649 \\
\end{array}$ \\
\hline
\end{tabular}

\begin{tabular}{|l|l|l|l|r||}
\hline \hline SOURCE & \multicolumn{1}{|c|}{ SITE } & \multicolumn{1}{|c|}{ GENUS1 } & \multicolumn{1}{|c|}{ SPECIES1 } & IDX1 \\
\hline DV & MESQUITE & Acinetobacter & calcoaceticus & 285 \\
DV & MESQUITE & Micrococcus & lylae sg B & 337 \\
DV & MESQUITE & No Match & & \\
DV & MESQUITE & Pseudomonas & pseudoalcaligenes & 410 \\
DV & MESQUITE & Pseudomonas & stutzeri & 67 \\
DV & MESQUITE & Pseudomonas & stutzeri & 228 \\
DV & MESQUITE & Pseudomonas & stutzeri & 242 \\
DV & MESQUITE & Pseudomonas & stutzeri & 289 \\
DV & MESQUITE & Pseudomonas & stutzeri & 332 \\
DV & MESQUITE & Pseudomonas & stutzeri & 375 \\
DV & MESQUITE & Pseudomonas & stutzeri & 390 \\
DV & MESQUITE & Pseudomonas & stutzeri & 478 \\
DV & MESQUITE & Pseudomonas & stutzeri & 512 \\
DV & MESQUITE & Pseudomonas & stutzeri & 524 \\
\hline
\end{tabular}

\begin{tabular}{|l|l|l|l|r||}
\hline SOURCE & \multicolumn{1}{|c|}{ SITE } & \multicolumn{1}{|c|}{ GENUS1 } & \multicolumn{1}{|c|}{ SPECIES1 } & IDX1 \\
\hline \hline DV & NAVARRES & Arthrobacter & pascens & 261 \\
DV & NAVARRES & Bacillus & amyloliquefaciens & 167 \\
DV & NAVARRES & Bacillus & macroides & 19 \\
DV & NAVARRES & Micrococcus & luteus sg A & 219 \\
DV & NAVARRES & Micrococcus & luteus sg A & 223 \\
DV & NAVARRES & Micrococcus & luteus sg A & 226 \\
DV & NAVARRES & Micrococcus & luteus sg A & 242 \\
DV & NAVARRES & Micrococcus & luteus sg A & 246 \\
DV & NAVARRES & Micrococcus & luteus sg A & 246 \\
DV & NAVARRES & Micrococcus & roseus & 211 \\
DV & NAVARRES & Micrococcus & roseus & 314 \\
DV & NAVARRES & Micrococcus & roseus & 326 \\
DV & NAVARRES & Micrococcus & roseus & \\
DV & NAVARRES & No Match & & \\
DV & NAVARRES & No Match & & \\
DV & NAVARRES & No Match & & \\
DV & NAVARRES & No Match & & \\
\hline
\end{tabular}




\begin{tabular}{|l|l|l|l|r|}
\hline SOURCE & \multicolumn{1}{|c|}{ SITE } & \multicolumn{1}{|c|}{ GENUS1 } & \multicolumn{1}{|c|}{ SPECIES1 } & IDX1 \\
\hline \hline DV & SCOTTY'S & Agrobacterium & radiobacter & 266 \\
DV & SCOTTY'S & Curtobacterium & flaccumfaciens poin & 540 \\
DV & SCOTTY'S & Cytophaga & aquatilis & 464 \\
DV & SCOTTY'S & Cytophaga & johnsonae & 249 \\
DV & SCOTTY'S & Micrococcus & luteus sg A & 201 \\
DV & SCOTTY'S & Micrococcus & luteus sg B & 507 \\
DV & SCOTTY'S & Micrococcus & lylae sg B & 136 \\
DV & SCOTTY'S & No Match & & \\
DV & SCOTTY'S & Pseur nmonas & syringae maculi & 431 \\
\hline
\end{tabular}

\begin{tabular}{|l|l|l|l|r|}
\hline SOURCE & \multicolumn{1}{|c|}{ SITE } & \multicolumn{1}{|c|}{ GENUS1 } & \multicolumn{1}{|c|}{ SPECIES1 } & IDX1 \\
\hline DV & TEXAS & Micrococcus & luteus sg A & 209 \\
DV & TEXAS & Micrococcus & lyloe sg B & 93 \\
DV & TEXAS & Micrococcus & roseus & 312 \\
DV & TEXAS & Micrococcus & roseus & 572 \\
DV & TEXAS & Micrococcus & roseus & 624 \\
DV & TEXAS & Micrococcus & roseus & 632 \\
DV & TEXAS & Micrococcus & roseus & 651 \\
DV & TEXAS & Micrococcus & roseus & 679 \\
DV & TEXAS & Micrococcus & roseus & 692 \\
DV & TEXAS & Micrococcus & roseus & 743 \\
DV & Micrococcus & roseus & 769 \\
DV & TEXAS & Micrococcus & roseus & 819 \\
DV & TEXAS & Micrococcus & roseus & \\
DV & TEXAS & NoXAS Match & & \\
\hline
\end{tabular}

\begin{tabular}{|c|c|c|c|c|}
\hline SOURCE & SITE. & GENUS1 & SPECIES 1 & $10 \times 1$ \\
\hline $\begin{array}{l}D V \\
D V \\
D V \\
D V \\
D V \\
D V \\
D V \\
D V \\
D V \\
D V \\
D V \\
D V \\
D V \\
D V \\
D V \\
D V \\
D V \\
D V \\
D V \\
\end{array}$ & \begin{tabular}{|l} 
TRAVA \\
TRAVA \\
TRAVA \\
TRAVA \\
TRAVA \\
TRAVA \\
TRAVA \\
TRAVA \\
TRAVA \\
TRAVA \\
TRAVA \\
TRAVA \\
TRAVA \\
TRAVA \\
TRAVA \\
TRAVA \\
TRAVA \\
TRAVA \\
TRAVA \\
\end{tabular} & $\begin{array}{l}\text { Hydrogenophaga } \\
\text { Micrococcus } \\
\text { Micrococcus } \\
\text { Micrococcus } \\
\text { Micrococcus } \\
\text { Micrococcus } \\
\text { Micrococcus } \\
\text { Micrococcus } \\
\text { No Match } \\
\text { Staphylococcus } \\
\text { Staphylococcus } \\
\text { Staphylococcus } \\
\text { Staphylococcus } \\
\text { Staphylococcus } \\
\text { Staphylococcus } \\
\text { Staphylococcus } \\
\text { Staphylococcus } \\
\text { Staphylococcus } \\
\text { Staphylococcus } \\
\end{array}$ & $\begin{array}{l}\text { palleronii } \\
\text { luteus sg A } \\
\text { luteus sg A } \\
\text { luteus sg A } \\
\text { roseus } \\
\text { roseus } \\
\text { roseus } \\
\text { roseus } \\
\text { epidermidis } \\
\text { epidermidis } \\
\text { epidermidis } \\
\text { epidermidis } \\
\text { epidermidis } \\
\text { epidermidis } \\
\text { epidermidis } \\
\text { epidermidis } \\
\text { warneri } \\
\text { warneri } \\
\end{array}$ & $\begin{array}{l}361 \\
110 \\
149 \\
198 \\
467 \\
526 \\
570 \\
662 \\
\\
707 \\
712 \\
723 \\
741 \\
755 \\
756 \\
760 \\
777 \\
780 \\
791 \\
\end{array}$ \\
\hline
\end{tabular}




\begin{tabular}{|l|l|l|l|r||}
\hline SOURCE & \multicolumn{1}{|c|}{ SITE } & \multicolumn{1}{|c|}{ GENUS1 } & \multicolumn{1}{|c|}{ SPECIES1 } & IDX1 \\
\hline DV & TRAVB & Micrococcus & kristinae & 379 \\
DV & TRAVB & Micrococcus & kristinae & 413 \\
DV & TRAVB & Micrococcus & luteus Sg A & 53 \\
DV & TRAVB & Micrococcus & roseus & 197 \\
DV & TRAVB & Micrococcus & roseus & 487 \\
DV & TRAVB & Micrococcus & roseus & 514 \\
DV & TRAVB & Micrococcus & roseus & 591 \\
DV & TRAVB & Micrococcus & roseus & 592 \\
DV & TRAVB & No Match & & \\
DV & TRAVB & No Match & & \\
DV & TRAVB & Match & \\
DV & TRAVB & No Match & \\
DV & TRAVB & No Match & & \\
DV & TRAVB & No Match & & \\
DV & TRAVB & No Match & \\
\hline
\end{tabular}


Table 4. Identitication of isolates from the Ash Meadows Wildlife Refuge.

\begin{tabular}{|l|l|l|l|r|}
\hline SOURCE & \multicolumn{1}{|c|}{ SITE } & \multicolumn{1}{|c|}{ GENUS1 } & \multicolumn{1}{|c|}{ SPECIES1 } & IDX1 \\
\hline AM & BRADFORD & Alteromonas & haloplanktis & haloplanktis \\
AM & BRADFORD & Alteromonas & haloris & 109 \\
AM & BRADFORD & Alteromonas & haloplanktis & 113 \\
AM & BRADFORD & No Match & & \\
AM & BRADFORD & No Match & & \\
AM & BRADFORD & No Match & & \\
AM & BRADFORD & No Match & & \\
AM & BRADFORD & No Match & & \\
AM & BRADFORD & No Match & & \\
AM & BRADFORD & No Match & & \\
AM & BRADFORD & Serratia & fonticola & \\
AM & BRADFORD & Sphingobacterium & multivorum & \\
AM & BRADFORD & Sphingobacterium & spiritivorum & 84 \\
\hline
\end{tabular}

\begin{tabular}{|l|l|l|l|r|}
\hline SOURCE & \multicolumn{1}{|c|}{ SITE } & \multicolumn{1}{|c|}{ GENUS1 } & \multicolumn{1}{|c|}{ SPECIES1 } & IDX1 \\
\hline \hline AM & COLD & Alteromonas & haloplanktis & 92 \\
AM & COLD & Arthrobacter & atrocyaneus & 465 \\
AM & COLD & Pseudomonas & vesicularis & 136 \\
\hline
\end{tabular}

\begin{tabular}{|c|c|c|c|c|}
\hline SOURCE & SITE & GENUS1 & SPECIES1 & IDX1 \\
\hline $\begin{array}{l}A M \\
A M\end{array}$ & $\begin{array}{l}\text { CRYSTAL } \\
\text { CRYSTAL }\end{array}$ & $\begin{array}{l}\text { Pseudomonas } \\
\text { Pseudomonas }\end{array}$ & $\begin{array}{l}\text { diminuta } \\
\text { vesicularis }\end{array}$ & $\begin{array}{r}33 \\
209 \\
\end{array}$ \\
\hline
\end{tabular}

\begin{tabular}{|l|l|l|l|r|}
\hline SOURCE & SITE & \multicolumn{1}{|c|}{ GENUS1 } & \multicolumn{1}{|c|}{ SPECIES1 } & IDX1 \\
\hline AM & DEVIL'S SURFAC & $\begin{array}{l}\text { Enterococcus } \\
\text { Hydrogenophaga }\end{array}$ & $\begin{array}{l}\text { gallinarum } \\
\text { pseudoflava }\end{array}$ & $\begin{array}{r}855 \\
\text { AM }\end{array}$ \\
\hline
\end{tabular}

\begin{tabular}{|l|l|l|l|r|}
\hline SOURCE & SITE & GENUS1 & SPECIES1 & IDX1 \\
\hline AM & DEVIL'S 20' & Pseudomonas & diminuta & 323 \\
AM & DEVIL'S 20' & Pseudomonas & diminuta & 880 \\
AM & DEVIL'S 20' & Pseudomonas & saccharophila & 403 \\
\hline
\end{tabular}

\begin{tabular}{|l|l|l|l|r|}
\hline SOURCE & \multicolumn{1}{|c|}{ SITE } & \multicolumn{1}{|c|}{ GENUS1 } & \multicolumn{1}{|c|}{ SPECIES1 } & IDX1 \\
\hline AM & DEVIL'S 40' & Clavibacter & michiganense in & 18 \\
AM & DEVIL'S 40' & Micrococcus & lyg B & 91 \\
diminuta & Pseudomonas & diminuta \\
\hline
\end{tabular}




\begin{tabular}{|l|l|l|l|r|}
\hline SOURCE & \multicolumn{1}{|c|}{ SITE } & \multicolumn{1}{|c|}{ GENUS1 } & \multicolumn{1}{|c|}{ SPECIES1 } & IDX1 \\
\hline \hline AM & DEVIL'S 85' & Acinetobacter & Iwoffii & 412 \\
AM & DEVIL'S 85' & Acinetobacter & Iwoffii & 572 \\
AM & DEVIL'S 85' & No Match & & \\
AM & DEVIL'S 85' & Pseudomonas & diminuta & 850 \\
AM & DEVIL'S 85' & Pseudomonas & diminuta & 864 \\
AM & DEVIL'S 85' & Pseudomonas & diminuta & 870 \\
AM & DEVIL'S 85' & Pseudomonas & saccharophila & 550 \\
AM & DEVIL'S 85' & Staphylococcus & hominis & 755 \\
AM & DEVIL'S 85' & Xanthomonas & maltophilia & 557 \\
\hline
\end{tabular}

\begin{tabular}{||l|l|l|l|r||}
\hline SOURCE & \multicolumn{1}{|c|}{ SITE } & \multicolumn{1}{|c|}{ SENUS1 } & \multicolumn{1}{|c|}{ SPECIES1 } & IDX1 \\
\hline AM & FOREST & Hydrogenophaga & pseudoflava & 80 \\
AM & FOREST & Hydrogenophaga & pseudoflava & 391 \\
AM & FOREST & Methylobacterium & zatmanii & 603 \\
AM & FOREST & Micrococcus & luteus sg A & 171 \\
AM & FOREST & Micrococcus & varians & 211 \\
AM & FOREST & No Match & & \\
AM & FOREST & No Match & & \\
AM & FOREST & No Match & & 163 \\
AM & FOREST & Pseudomonas & vesicularis & 684 \\
AM & FOREST & Pseudomonas & vesicularis & \\
\hline
\end{tabular}

\begin{tabular}{|l|l|l|l|l|}
\hline SOURCE & SITE & GENUS1 & SPECIES1 & IDX1 \\
\hline AM & INDIAN & Rhodococcus & equi sg B & 205 \\
\hline
\end{tabular}

\begin{tabular}{|l|l|l|l|r|}
\hline SOURCE & \multicolumn{1}{|c|}{ SITE } & \multicolumn{1}{|c|}{ GENUS1 } & \multicolumn{1}{|c|}{ SPECIES1 } & IDX1 \\
\hline AM & KINGSPOOL & Acidovorax & facilis & 847 \\
AM & KINGSPOOL & Alcaligenes & piechaudii & 173 \\
AM & KINGSPOOL & Alteromonas & haloplanktis & 23 \\
AM & KINGSPOOL & Alteromonas & haloplanktis & 27 \\
AM & KINGSPOOL & Alteromonas & haloplanktis & 140 \\
AM & KINGSPOOL & Alteromonas & haloplanktis & 220 \\
AM & KINGSPOOL & Alteromonas & haloplanktis & 231 \\
AM & KINGSPOOL & Alteromonas & haloplankís & 235 \\
AM & KINGSPOOL & No Match & & \\
AM & KINGSPOOL & No Match & & \\
AM & KINGSPOOL & No Match & & \\
AM & KINGSPOOL & No Match & & \\
AM & KINGSPOOL & No Match & & \\
AM & KINGSPOOL & Pseudomonas & pseudoalcaligenes & 215 \\
AM & KINGSPOOL & Pseudomonas & pseudoalcaligenes & 307 \\
AM & KINGSPOOL & Sphingobacterium & multivorum & 41 \\
AM & KINGSPOOL & Weeksella & virosa & 45 \\
\hline
\end{tabular}




\begin{tabular}{|l|l|l|l|r|}
\hline SOURCE & \multicolumn{1}{|c|}{ SITE } & \multicolumn{1}{|c|}{ GENUS1 } & \multicolumn{1}{|c|}{ SPECIES1 } & IDX1 \\
\hline AM & LONGSTREET & Aeromonas & hydrophila & 778 \\
AM & LONGSTREET & Bacillus & coagulans sg B & 222 \\
AM & LONGSTREET & Cytophaga & johnsonae & 14 \\
AM & LONGSTREET & Cytophaga & johnsonae & 57 \\
$A M$ & LONGSTREET & Enterobacter & agglomerans & 360 \\
$A M$ & LONGSTREET & Enterobacter & agglomerans & 395 \\
$A M$ & LONGSTREET & Enterobacter & agglomerans & 431 \\
$A M$ & LONGSTREET & Methylobacterium & zatmanii & 472 \\
$A M$ & LONGSTREET & Shewanella & putrefaciens & 50 \\
AM & LONGSTREET & Xanthobacter & flavus & 307 \\
\hline
\end{tabular}

\begin{tabular}{|l|l|l|l|r|}
\hline SOURCE & SITE & GENUS1 & SPECIES1 & IDX1 \\
\hline \hline $\begin{array}{l}\text { AM } \\
\text { AM }\end{array}$ & $\begin{array}{l}\text { MARSH } \\
\text { MARSH }\end{array}$ & $\begin{array}{l}\text { Flavobacterium } \\
\text { No Match }\end{array}$ & resinovorum & 460 \\
\hline
\end{tabular}

\begin{tabular}{|c|c|c|c|c|}
\hline SOURCE & SITE & GENUS1 & SPECIES1 & IDX1 \\
\hline $\begin{array}{l}A M \\
A M\end{array}$ & $\begin{array}{l}\text { ROGERS } \\
\text { ROGERS }\end{array}$ & $\begin{array}{l}\text { Listonella } \\
\text { Pseudomonas }\end{array}$ & $\begin{array}{l}\text { anguillarum } \\
\text { coronafaciens }\end{array}$ & $\begin{array}{l}161 \\
388\end{array}$ \\
\hline
\end{tabular}

\begin{tabular}{|l|l|l|l|r|}
\hline SOURCE & \multicolumn{1}{|c|}{ SITE } & \multicolumn{1}{|c|}{ GENUS1 } & \multicolumn{1}{|c|}{ SPECIES1 } & IDX1 \\
\hline \hline AM & SCHOOL1 & Alteromonas & haloplanktis & 120 \\
AM & SCHOOL1 & Alteromonas & haloplanktis & 251 \\
AM & SCHOOL1 & Arthrobacter & atrocyaneus & 450 \\
AM & SCHOOL1 & Chromobacterium & violaceum & 537 \\
AM & SCHOOL1 & Chromobacterium & violaceum & 576 \\
AM & SCHOOL1 & Micrococcus & luteus sg A & 482 \\
AM & SCHOOL1 & No Match & & \\
AM & SCHOOL1 & Pseudomonas & alcaligenes & 241 \\
AM & SCHOOL1 & Pseudomonas & coronafaciens & 252 \\
AM & SCHOOL1 & Pseudomonas & pseudoalcaligenes & 200 \\
AM & SCHOOL1 & Pseudomonas & stutzeri & 663 \\
AM & SCHOOL1 & Pseudomonas & stutzeri & 776 \\
AM & SCHOOL1 & Pseudomonas & stutzeri & 838 \\
\hline
\end{tabular}




\begin{tabular}{|l|l|l|l|r|}
\hline SOURCE & \multicolumn{1}{|c|}{ SITE } & \multicolumn{1}{|c|}{ GENUS1 } & \multicolumn{1}{|c|}{ SPECIES1 } & IDX1 \\
\hline AM & SCHOOL2 & Chromobacterium & violaceum & 498 \\
AM & SCHOOL2 & Chromobacterium & violaceum & 563 \\
AM & SCHOOL2 & No Match & & \\
AM & SCHOOL2 & No Match & & \\
AM & SCHOOL2 & Pseudomonas & alcaligenes & 629 \\
AM & SCHOOL2 & Pseudomonas & coronafaciens & 533 \\
AM & SCHOOL2 & Pseudomonas & coronafaciens & 578 \\
AM & SCHOOL2 & Pseudomonas & stutzeri & 872 \\
AM & SCHOOL2 & Pseudomonas & Stutzeri & 889 \\
AM & SCHOOL2 & Pseudomonas & stutzeri & 922 \\
AM & SCHOOL2 & Pseudomonas & syringae maculi & 542 \\
\hline
\end{tabular}

\begin{tabular}{|l|l|l|l|r|}
\hline SOURCE & \multicolumn{1}{|c|}{ SITE } & \multicolumn{1}{|c|}{ GENUS1 } & \multicolumn{1}{|c|}{ SPECIES1 } & IDX1 \\
\hline AM & SCRUGGS & Agrobacterium & radiobacter & 426 \\
AM & SCRUGGS & Micrococcus & luteus Sg A & 32 \\
AM & SCRUGGS & Micrococcus & lylae sg B & 27 \\
AM & SCRUGGS & No Match & Saucimobilis & 73 \\
AM & SCRUGGS & Sphingomonas & paucimobomis \\
\hline
\end{tabular}

\begin{tabular}{|l|l|l|l|r|}
\hline SOURCE & \multicolumn{1}{|c|}{ SITE } & \multicolumn{1}{|c|}{ GENUS1 } & \multicolumn{1}{|c|}{ SPECIES1 } & IDX1 \\
\hline AM & SWROCKS & No Match & & \\
AM & SW ROCKS & Pseudomonas & vesicularis \\
AMM & SW ROCKalis & 158 \\
\hline
\end{tabular}

\begin{tabular}{|l|l|l|l|r|}
\hline SOURCE & \multicolumn{1}{|c|}{ SITE } & \multicolumn{1}{|c|}{ GENUS1 } & \multicolumn{1}{|c|}{ SPECIES1 } & IDX1 \\
\hline AM & TUBBS1 & Alteromonas & haloplanktis & 86 \\
AM & TUBBS1 & Ctyophaga & johnsonae & 98 \\
AM & TUBBS1 & No Match & & \\
AM & TUBBS1 & No Match & & \\
AM & TUBBS1 & No Match & & \\
AM & TUBBS1 & Pseudomonas & syringae maculi & 913 \\
AM & TUBS1 & Pseudomonas & syringae maculi & 913 \\
\hline
\end{tabular}

\begin{tabular}{|c|c|c|c|c|}
\hline SOURCE & SITE & GENUS1 & SPECIES1 & IDX1 \\
\hline $\begin{array}{l}A M \\
A M \\
A M \\
A M \\
A M\end{array}$ & $\begin{array}{l}\text { TUBBS2 } \\
\text { TUBBS2 } \\
\text { TUBBS2 } \\
\text { TUBBS2 } \\
\text { TUBBS2 }\end{array}$ & $\begin{array}{l}\text { Curtobacterium } \\
\text { Curtobacterium } \\
\text { No Match } \\
\text { Pseudomonas } \\
\text { Staphylococcus } \\
\end{array}$ & $\begin{array}{l}\text { flaccumfacien } f \\
\text { flaccumfacien poin } \\
\text { syringae maculi } \\
\text { xylosus }\end{array}$ & $\begin{array}{r}33 \\
719 \\
\\
178 \\
141 \\
\end{array}$ \\
\hline
\end{tabular}



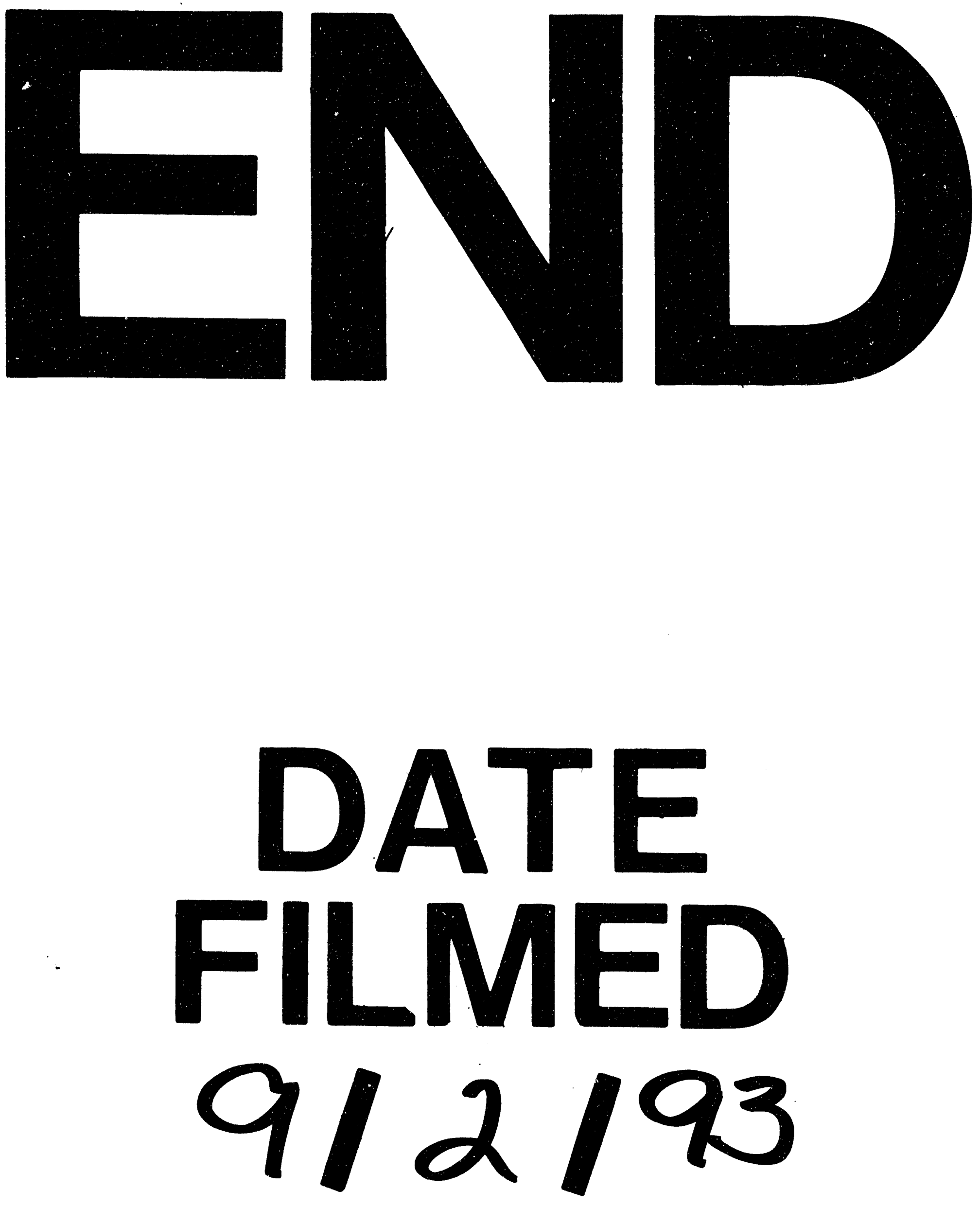
. 